\title{
Lamb Temporal Bone as a Surgical Training Model of Round Window Cochlear Implant Electrode Insertion
}

\author{
*Georgios Mantokoudis, *Markus E. Huth, †Christian Weisstanner, *Hergen M. Friedrich, \\ $\dagger$ †laude Nauer, *Claudia Candreia, *Marco D. Caversaccio, and *Pascal Senn \\ *University Department of Otorhinolaryngology, Head and Neck Surgery; and †University Department of Neuroradiology and \\ Interventional Radiology, Inselspital Bern, Switzerland
}

Objective: The preservation of residual hearing in cochlear implantation opens the door for optimal functional results. This atraumatic surgical technique requires training; however, the traditional human cadaveric temporal bones have become less available or unattainable in some institutions. This study investigates the suitability of an alternative model, using cadaveric lamb temporal bone, for surgical training of atraumatic round window electrode insertion.

Intervention: A total of 14 lamb temporal bones were dissected for cochlear implantation by four surgeons. After mastoidectomy, visualization, and drilling of the round window niche, an atraumatic round window insertion of a Medel Flex24 electrode was performed. Electrode insertion depth and position were verified by computed tomography scans.

Main Outcome Measure: All cochleas were successfully implanted using the atraumatic round window approach; however, surgical access through the mastoid was substantially different when compared human anatomy. The mean number of intracochlear electrode contacts was 6.5 (range, 4-11) and the mean insertion depth $10.4 \mathrm{~mm}$ (range, 4-20 mm), which corresponds to a mean angular perimodiolar insertion depth of 229 degrees (range 67$540^{\circ}$ ). Full insertion of the electrode was not possible because of the smaller size of the lamb cochlea in comparison to that of the human.

Conclusion: The lamb temporal bone model is well suited as a training model for atraumatic cochlear implantation at the level of the round window. The minimally pneumatized mastoid as well as the smaller cochlea can help prepare a surgeon for difficult cochlear implantations. Because of substantial differences to human anatomy, it is not an adequate training model for other surgical techniques such as mastoidectomy and posterior tympanotomy as well as full electrode insertion. Key Words: Animal model-Cochlear implantation-Electrode insertion-Lamb-SheepTemporal bone.

Otol Neurotol 37:52-56, 2016.
Cochlear implantation (CI) with hearing preservation is a challenging low-volume procedure (1) and thus requires surgical training. The gold standard for CI training uses human cadaveric temporal bones (HTB) (2). As the most realistic model, HTBs provide the proper conditions for improving hand-eye coordination, fine motor skills, and anatomical knowledge under microscopically magnified vision (2). However, the availability of HTBs is limited because of legal, economical, and ethical

Address correspondence and reprint requests to Dr. med. Markus E. Huth, University Department of Otorhinolaryngology, Head and Neck Surgery, Inselspital, 3010 Bern, Switzerland; E-mail: markus.huth@ insel.ch

Georgios Mantokoudis and Markus E. Huth equally contributed to this work. This work was financed by internal funding from the University Department of Otorhinolaryngology, Head and Neck Surgery at Inselspital Bern.

The authors disclose no conflicts of interest. reasons (2,3). Artificial temporal bones provide a three-dimensional training opportunity for anatomy visualization, but the tissue morphology is unnatural (4-6). Similarly, computer-based simulation models provide valuable anatomical training (7) and 3D virtual reality simulations are convincing but still cannot produce natural tissue morphology (8).

This study defines the utilization of the lamb temporal bone (LTB) as a surgical training model for CI. This study particularly emphasizes the training of round window niche (RWN) preparation with 1) drill removal of the bone overhang without damaging the membrane, 2) removal of mucosal folds (pseudomembranes), and 3) vertical incision of the round window membrane (RWM). The overall training goal is to maximize electrode insertion while minimizing intracochlear trauma. Furthermore, the usability of the LTB as a model for training intracochlear electrode insertion is quantified and evaluated. 


\section{INTERVENTION}

\section{Preparations}

Seven lamb heads (9-12-mo-old) were acquired from a regional slaughterhouse. After skin removal, all heads were fixed in $4 \%$ formaldehyde ( $\mathrm{pH} 7.4$ ) for 2 weeks. For dissection the LTB were positioned in a drilling shell in the lateral position (Fig. 1). Soft tissues around the external auditory canal (EAC) and the retroauricular space were
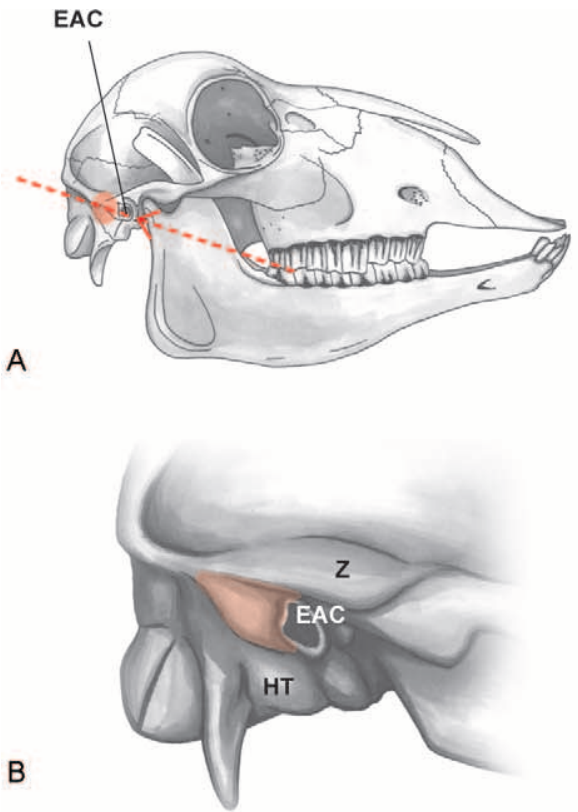

C

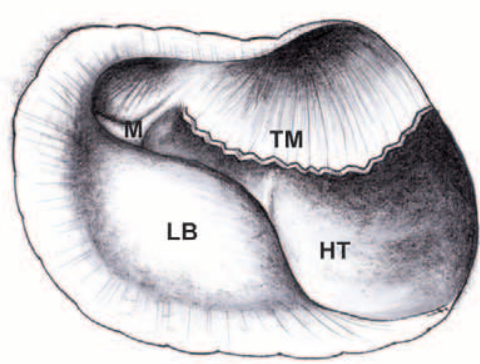

D

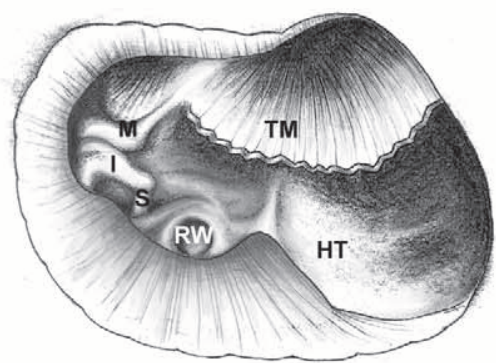

removed to expose the mastoidal plane. Landmarks such as the temporal line, the mastoid tip, and the zygoma were identified (Fig. 1, A, B, and E). Four otolaryngology (ENT) surgeons with no, low, moderate, and advanced experience levels participated in the study.

\section{Surgical Approach}

A canal-wall-down mastoidectomy of the nonpneumatized mastoid was performed (Fig. 1). The mastoid
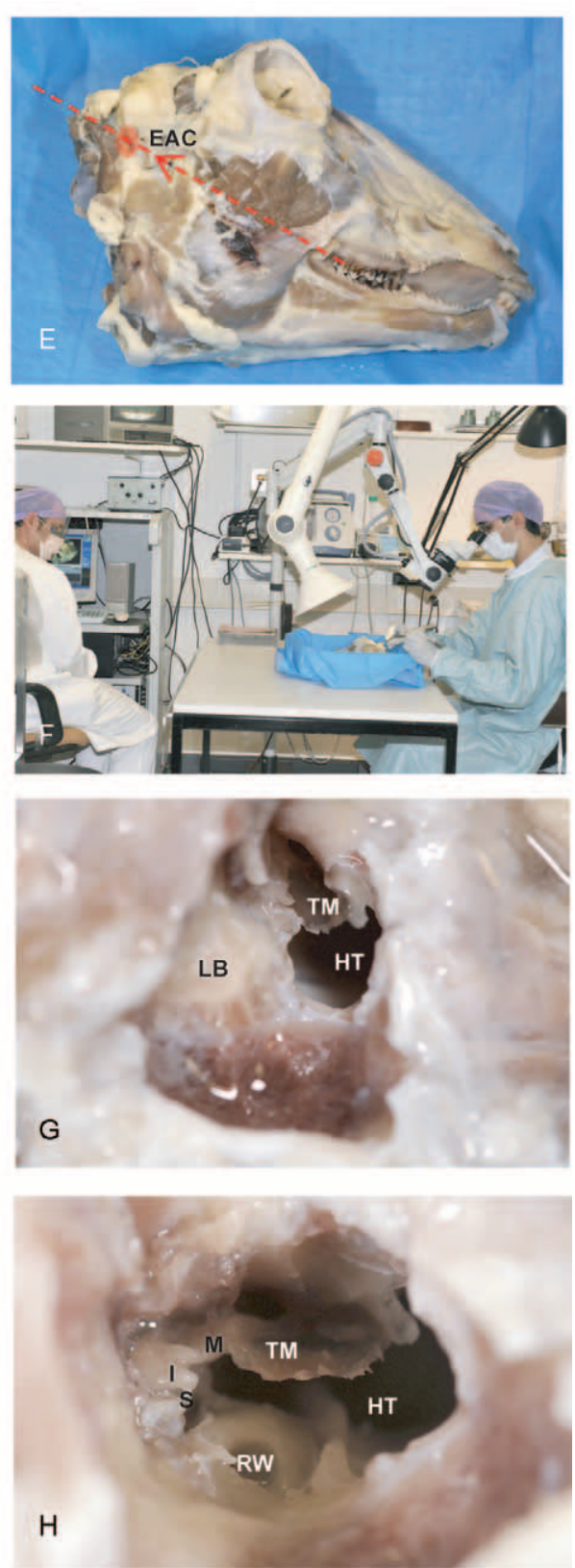

FIG. 1. Overview of surgical landmarks on the sheep skull $(A, E)$, in the mastoid $(C, G)$, and in the middle ear $(D, H)$. The imaginary line between the occlusion plane of teeth and the lowest point of the bony external ear canal indicates the position of the round window $(A, E)$. The round window niche is exposed after removal of the eminentia pyramidalis and part of the labyrinth block together with the facial nerve $(D, H)$. The skull should be positioned in the lateral decubitus position with the mouth facing away from the surgeon $(E)$. A conventional (human) temporal bone laboratory is well suited for the procedure, particularly if lamb heads are used $(F)$. EAC indicates external auditory canal; H, hypotympanon; I, incus; LB, labyrinth block; M, malleus; S, stapes; TM, tympanic membrane. 
was drilled down to the osseous labyrinth block (LB) (Fig. 1, C and G). The bullous hypotympanon was opened inferiorly to the LB. The malleus and incus were readily identified (Fig. 1, D and H), whereas the stapes was only partially visible. The border of the LB toward the middle ear was drilled down until the stapedial muscle appeared. Further drilling of the eminentia pyramidalis with a smaller Rosen burr exposed the RWN completely. Understanding that this technique is not possible in humans, the facial nerve and the LB were partially removed to optimize the view into the RWN. The round window (RW) is situated in an extended imaginary line between the plane of teeth occlusion and the lowest point of the bony EAC (dotted line in Fig. 1A). Because of the different mastoid and middle ear anatomy compared with humans, a classical posterior tympanotomy could not be performed in the lamb.

\section{Round Window Approach and Electrode Array Insertion}

The overhanging bone of the RWN was completely removed using small diamond drills of 1 to $1.5 \mathrm{~mm}$ with a primary goal of preventing damage to the RWM. Any pseudomembranes covering the RWN were removed using microhooks. With particular emphasis on the atraumatic technique, the RWM was vertically incised using a small tympanotomy knife. Finally, the electrode array was inserted through the RWM using specially designed claws or alligator forceps (Fig. 2). Each of the surgeons was asked to insert the maximum number of electrode contacts possible, while aiming for minimal trauma of intracochlear structures.

For all experiments, flex24 electrode arrays (MED-EL, Innsbruck, Austria) were used. These human-grade arrays had failed internal quality controls in the company and were shipped to us without the implant stimulator unit for research purposes. Each array with a length of $24 \mathrm{~mm}$ and a basal diameter of $0.8 \mathrm{~mm}$ features 12 electrode contacts, each with dimensions of $800 \mu \mathrm{m} \times 500 \mu \mathrm{m}$, spaced $1.9 \mathrm{~mm}$ apart. This electrode was specifically designed for electro-acoustical stimulation with an emphasis on atraumatic implantation and hearing preservation (22).

\section{Control of Electrode Position by CT Scan}

All LTB were scanned immediately after implantation using a clinical spiral CT scanner (Lightspeed Ultra, GE Healthcare, Milwaukee, WI, U.S.A.). A high-resolution CT using a standard clinical protocol (coronal plane, field of view $10 \mathrm{~cm}$ ) with $140 \mathrm{kV}$ and $65 \mathrm{~mA}$ was performed. A standard bone plus reconstruction kernel (reconstruction algorithm) with $68 \%$ overlapping section thickness of $0.625 \mathrm{~mm}$ was used.

\section{MAIN OUTCOME MEASURE}

\section{Surgical Evaluation}

The required dissection time was measured, and the surgical performance was self-evaluated by each surgeon regarding the mastoidectomy, round window approach, and electrode insertion.

\section{Radiologic Evaluation}

Electrode insertion was further objectified radiologically. The electrode position, the number of intracochlear electrode contacts, and angular insertion depths were evaluated by a senior staff radiologist on a standard workstation (Easyvision, Philips, Hamburg, Germany).

\section{RESULTS}

\section{Mastoidectomy}

The exposure of the RW by canal-wall-down mastoidectomy was successful in all attempts, with a mean dissection time of 31 minutes (range, 20-50 min). All surgeons demonstrated a decreased dissection time of 5 to 10 minutes with repeated procedures. The initial
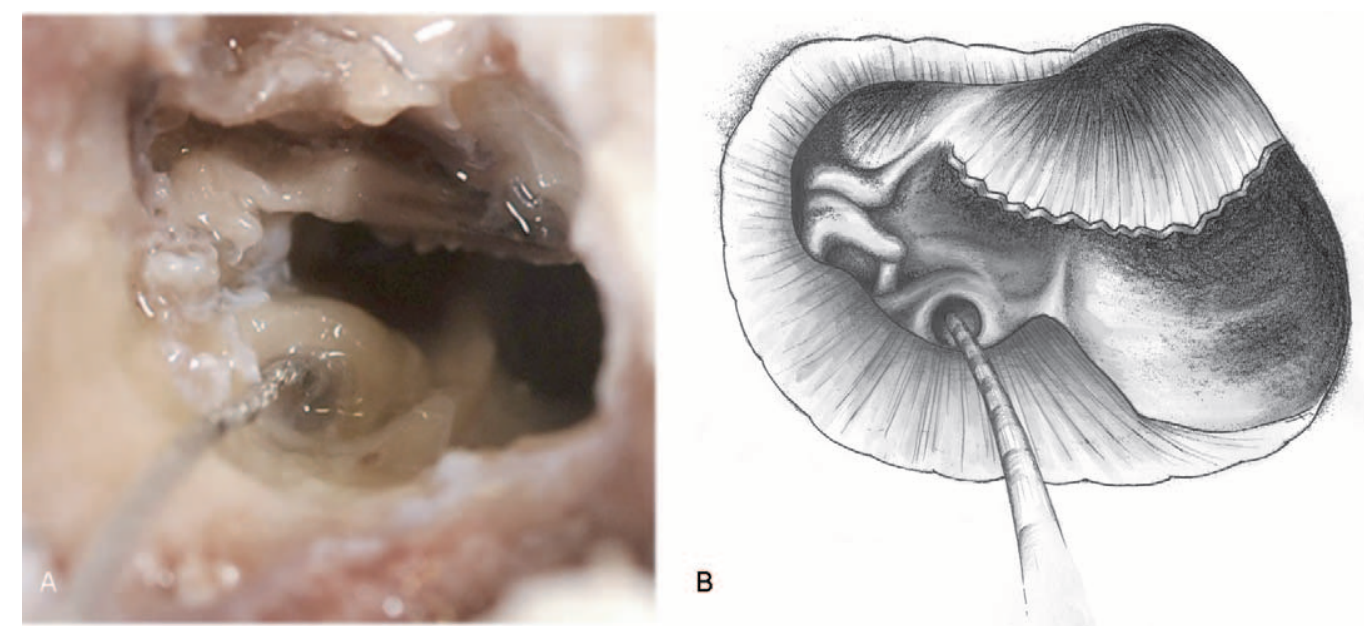

FIG. 2. Carefully prepared round window niche with flex24 electrode array atraumatically inserted through the incised round window membrane.

Otology \& Neurotology, Vol. 37, No. 1, 2016 


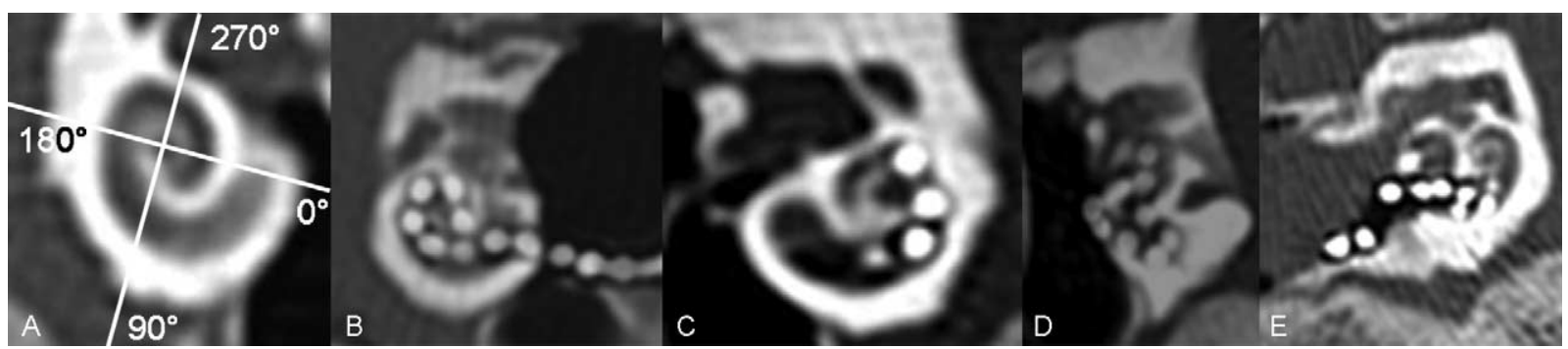

FIG. 3. Examples of sheep temporal bone CT scans for angular insertion depths $(A)$ and different intracochlear electrode positions: maximum insertion in scala tympani $(B)$, incomplete insertion $(C)$, malpositioned array in the utricle $(D)$, and intracochlear folding $(E)$.

dissection of at least one LTB was required to learn the anatomy and surgical access technique via mastoidectomy. The lamb mastoid is minimally pneumatized and the sigmoid sinus is not in the vicinity to serve as a landmark. Usable anatomical landmarks are the posterior wall of the EAC and the suprameatal (Henle's) spine. The EAC diameter is smaller in the lamb but demonstrates the same axial orientation as in a human. The posterior wall of the lamb EAC has to be completely removed to allow for complete middle ear exposure. The LB was more difficult to identify because of the smaller size and the less pronounced yellowish bone color.

\section{Round Window Approach}

The RWN was always accessible through the proposed approach. The size of the niche was comparable to human anatomy. The three critical tasks of 1) drilling the RWN without damaging the membrane, 2) removal of pseudomembranes, and 3) vertical incision of the RWM were successfully achieved by all surgeons. There was no malformation or anatomical abnormality observed in any of the 14 dissected LTBs.

\section{Electrode Insertion}

The task of maximizing electrode insertion while minimizing intracochlear trauma was perceived as being challenging, particularly by the surgeons with little to no experience. The mean surgical insertion depth was $10.4 \mathrm{~mm}$ (range, $4-20 \mathrm{~mm}$ ), and the mean radiological angular insertion depth was 229 degrees (range, 67$540^{\circ}$ ) around the modiolus. Figure $3 \mathrm{~A}$ shows the angular definitions and Table 1 the electrode insertion depth for each LTB and for each surgeon. The mean number of electrode contacts inserted was 6.5 (range, 4-11); full insertion of 12 electrode contacts was never achieved. A correlation between insertion depths and the experience of the surgeon was observed; however, because of the limited number of insertions per surgeon, statistical relevance cannot be determined (Table 1). Furthermore, intracochlear array deformation occurred in a total of three $(21 \%)$ implantations, of which one occurred in the hands of the most experienced surgeon. In addition, half of the trials did terminate in the scala tympani, as six electrodes deviated from the scala tympani to the scala vestibuli (scale shift); one electrode array ended up in the inner ear vestibule (Table 1, Fig. 3D).

TABLE 1. Evaluation of CI electrode positions and insertion depths after implantation by four ENT surgeons with different levels of otologic surgical experience

\begin{tabular}{|c|c|c|c|c|c|c|c|}
\hline \multirow[b]{2}{*}{ Experience Level } & \multirow[b]{2}{*}{ \# } & \multirow[b]{2}{*}{ Time $(\min )$} & \multicolumn{2}{|c|}{ Insertion Depth } & \multirow[b]{2}{*}{ Intracochlear Electrodes } & \multicolumn{2}{|c|}{$\begin{array}{l}\text { Intracochlear } \\
\text { Electrode Course }\end{array}$} \\
\hline & & & $(\mathrm{mm})$ & $\left({ }^{\circ}\right)$ & & & \\
\hline \multirow[t]{3}{*}{ No experience } & 1 & 35 & 6 & 180 & 4 & Normal & ST \\
\hline & 2 & 30 & 12 & 67 & 4 & Misplaced & $\mathrm{U}$ \\
\hline & 3 & 20 & 4 & 172 & 4 & Normal & $\mathrm{ST}$ \\
\hline \multirow[t]{7}{*}{ Beginner } & 1 & 50 & 13 & 165 & 8 & Folded & $\mathrm{ST} \rightarrow \mathrm{SV}$ \\
\hline & 2 & 40 & 6 & 217 & 4 & Normal & ST \\
\hline & 3 & 30 & 11 & 173 & 7 & Scale shift & $\mathrm{ST} \rightarrow \mathrm{SV}$ \\
\hline & 4 & 20 & 8 & 220 & 5 & Normal & ST \\
\hline & 5 & 30 & 8 & 140 & 5 & Folded & $\mathrm{ST} \rightarrow \mathrm{SV}$ \\
\hline & 6 & 30 & 11 & 273 & 8 & Scale shift & $\mathrm{ST} \rightarrow \mathrm{SV}$ \\
\hline & 7 & 20 & 6 & 185 & 5 & Scale shift & $\mathrm{ST} \rightarrow \mathrm{SV}$ \\
\hline \multirow[t]{2}{*}{ Moderate } & 1 & 25 & 17 & 540 & 11 & Normal & ST \\
\hline & 2 & 20 & 8 & 234 & 6 & Normal & $\mathrm{ST}$ \\
\hline \multirow[t]{2}{*}{ Advanced } & 1 & 40 & 20 & 205 & 11 & Folded & $\mathrm{ST} \rightarrow \mathrm{SV}$ \\
\hline & 2 & 20 & 15 & 431 & 9 & Normal & ST \\
\hline
\end{tabular}

The implantations are ordered in sequence (\#) for each surgeon to allow for assessing the learning effects. ST indicates scala tympani; SV, scala vestibuli; $U$, utricle. 


\section{CONCLUSIONS}

The LTB is considerably different compared with human anatomy and is therefore unlikely to completely replace HTBs for ear surgery training. However, at the level of the RWN the wide visualization allows for training of the delicate drilling of the bony overhang of the niche, the removal of pseudomembranes without damaging the membrane, and the controlled incision of the RWM. Full electrode insertion could not be achieved and, even in the case of reasonable insertion depths, intracochlear array deformation and scale shifts were observed independently of the surgeon's experience. Therefore, the LTB provides an adequate model to train at the level of the RWN, whereas the minimally pneumatized mastoid as well as the smaller cochlea can help prepare a surgeon for difficult CIs.

Acknowledgments: The authors thank the MEDEL corporation for providing the cochlear implant electrodes and Catherine Reid for language editing the manuscript.

\section{REFERENCES}

1. Taylor F, Hine C. Cochlear implants: Informing commissioning decisions, based on need. J Laryngol Otol 2006;120:1008-13.

2. George AP, De R. Review of temporal bone dissection teaching: How it was, is and will be. J Laryngol Otol 2010;124:119-25.

3. Campbell CS. Research on human tissue: religious perspectives. In: Commission NBA, editor. Research Involving Human Biological Materials: Ethical Issues and Policy Guidance-Commisioned Papers. Rockland, MD: National Bioethics Advisory Commission; 2000. pp. 1-22.

4. Mori K, Yamamoto T, Oyama K, et al. Modified three-dimensional skull base model with artificial dura mater, cranial nerves, and venous sinuses for training in skull base surgery: Technical note. Neurol Med Chir (Tokyo) 2008;48:582-7.

5. Schwager K, Gilyoma JM. Ceramic model for temporal bone exercises: An alternative for human temporal bones? Laryngorhinootologie 2003;82:683-6.

6. Suzuki M, Ogawa Y, Hagiwara A, et al. Rapidly prototyped temporal bone model for otological education. ORL J Otorhinolaryngol Relat Spec 2004;66:62-4.

7. Sorensen MS, Mosegaard J, Trier P. The visible ear simulator: A public PC application for GPU-accelerated haptic 3D simulation of ear surgery based on the visible ear data. Otol Neurotol 2009;30:484-7.

8. Morris D, Sewell C, Barbagli F, et al. Visuohaptic simulation of bone surgery for training and evaluation. IEEE Comput Graph Appl 2006;26:48-57. 